\title{
Feeding ducks, bacterial chemotaxis, and the Gini index
}

\author{
François J. Peaudecerf and Raymond E. Goldstein \\ Department of Applied Mathematics and Theoretical Physics, Centre for Mathematical Sciences, University of Cambridge, Wilberforce Road, \\ Cambridge CB3 OWA, United Kingdom
}

(Received 8 December 2014; revised manuscript received 16 June 2015; published 5 August 2015)

\begin{abstract}
Classic experiments on the distribution of ducks around separated food sources found consistency with the "ideal free" distribution in which the local population is proportional to the local supply rate. Motivated by this experiment and others, we examine the analogous problem in the microbial world: the distribution of chemotactic bacteria around multiple nearby food sources. In contrast to the optimization of uptake rate that may hold at the level of a single cell in a spatially varying nutrient field, nutrient consumption by a population of chemotactic cells will modify the nutrient field, and the uptake rate will generally vary throughout the population. Through a simple model we study the distribution of resource uptake in the presence of chemotaxis, consumption, and diffusion of both bacteria and nutrients. Borrowing from the field of theoretical economics, we explore how the Gini index can be used as a means to quantify the inequalities of uptake. The redistributive effect of chemotaxis can lead to a phenomenon we term "chemotactic levelling," and the influence of these results on population fitness are briefly considered.
\end{abstract}

DOI: 10.1103/PhysRevE.92.022701

PACS number(s): 87.18.Gh, 87.17.Jj, 87.23.-n, 89.65.-s

\section{INTRODUCTION}

In one of the more amusing, yet influential experiments on animal behavior, Harper [1] studied the distribution of mallards around two separated sources of standardized pieces of bread. After an induction period on the order of a minute, the average number of ducks clustered tightly around each station stabilized. The distribution he observed was simple: the number of ducks at each source was proportional to the flux of bread there (pieces/minute). This constituted the first experimental observation of the so-called ideal free distribution previously introduced in theoretical ecology. Using the terminology of Fretwell and Lucas [2], "ideal" means that ducks can identify the source where their uptake is maximized, and "free" implies unfettered ability to access the source of choice. This distribution, resulting from individual rational behaviors, achieves a population-wide uniformization of the probability of uptake and can be understood as a Nash equilibrium [3]. These works impacted not only ornithology, but ecology [4,5], evolutionary biology [6], and the study of human behavior [7-9], all areas involving resource acquisition in a heterogeneous environment.

Here we take motivation from Harper's experiment, and others discussed below, to examine resource acquisition in a heterogeneous microbial world [10], where swimming microorganisms respond to nutrient sources through concentration fields determined by molecular diffusion and microbial uptake. For the specific case of peritrichously flagellated bacteria such as Escherichia coli and Bacillus subtilis, cells move in a run-and-tumble random walk biased by concentration gradients, resulting in drift of the population up these gradients [11]. Because chemotaxis [12] is quite different

Published by the American Physical Society under the terms of the Creative Commons Attribution 3.0 License. Further distribution of this work must maintain attribution to the author(s) and the published article's title, journal citation, and DOI. from the visually based searching of higher animals, and because of the diffusive behavior of nutrients and the cell populations, the microbial problem is distinct in character. This feature motivates the present investigation of the consequences of a collection of individual chemotactic responses on the population-scale distribution of resources. While chemotaxis is generally thought to optimize uptake at the single-cell level [13], even the mere presence of translational diffusion in a population would lead to a distribution of uptake rates. And the interplay of chemotaxis and consumption will modify the distribution of resources and cells, with further potential impact on the uptake rate distribution. In this paper we focus on three key questions in this area: What is the distribution of bacteria around spatially distinct nutrient sources and their associated impact on the resource field? What is the distribution of resource uptake rates within that population? What are the consequences of such distributions for cellular fitness?

A historically important experiment on spatially varying resources is Engelmann's 1883 determination of the action spectrum of photosynthesis [14]. Having discovered bacteria that are attracted to the oxygen produced by photosynthesis [15], he imaged the solar spectrum onto a linear algal cell in an airtight chamber containing such bacteria. They clustered around the alga in proportion to the local oxygen production, revealing with greater precision than the available techniques of the time the peaks of photosynthetic activity for blue and red wavelengths. How reliably the local bacterial accumulation reflects the oxygen production rate, in the face of both bacterial and oxygen diffusion, remains an open question in the spirit of the present investigation. Moreover, this work demonstrates how microdomains releasing a limited quantity of attractive nutrients in a continuous fashion can arise in the microbial world.

Understanding bacterial organization and uptake around algal resources also finds an important biological context in the case of bacterial-algal symbiosis. The recent discovery [16] that many algae dependent on vitamin B12 obtain it through symbiotic relationships with bacteria raises questions about 
spatiotemporal aspects of symbiosis: how the two species find each other and arrange themselves to achieve the symbiosis.

These are examples of a more general problem of microorganisms responding to the "patchy" nature of nutrients in ecosystems [17], such as the prosaically named "marine snow" [18]. We emphasize the fundamental difference between live microbial sources, such as Engelmann's alga, and inert sources, such as lysis events; the former continuously release nutrients at low rates. They are thus stable in time but can be significantly impacted by bacterial uptake. These characteristics makes them the natural microbial equivalent to the limited continuous sources considered by Harper in his animal experiments.

To make concrete the interplay between production, consumption, diffusion, and chemotaxis we consider a generalized Keller-Segel (KS) model [19]. While the KS model is a well-established model that has found frequent application in the study of spatially extended microorganism populations [20,21], the biological setting of localized, low-intensity sources with a steady release of nutrients is little-studied, and the overarching issue of resource uptake distribution is essentially unexplored. In what follows the steady-state distributions arising from multiple localized sources are analyzed to understand the consequences of chemotaxis on the distribution of uptake in the bacterial population, the total uptake being fixed. Borrowing from theoretical economics, we next propose that the Gini index [22], a number originally used to characterize wealth inequality, can be used to quantify the individual uptake distribution. Varying the model parameters and the dimensionality of space we show that the combination of screening effects [23] and chemotaxis can switch from redistributing the resource to generating greater inequalities. Finally, we explore the potential biological consequences of uptake redistribution through an example of growth at low nutrient levels.

\section{THE MODEL}

Consider bacteria with mean concentration $b_{0}$ and local concentration $b(\mathbf{r}, t)$ in a $d$-dimensional volume $L^{d}$. Within the volume are nutrient sources with fluxes $\left\{\phi_{i}\right\}$ of typical value $\phi_{0}$ leading to a concentration field $c(\mathbf{r}, t) . b$ and $c$ obey KS equations [19],

$$
\begin{gathered}
\frac{\partial c}{\partial t}=D_{c} \nabla^{2} c-b f(c), \\
\frac{\partial b}{\partial t}=D_{b} \nabla^{2} b-\nabla \cdot[\chi(c) b \nabla c],
\end{gathered}
$$

with $D_{c}$ and $D_{b}$ the diffusion coefficients of nutrients and bacteria, respectively, and $\chi(c)$ the chemotactic response coefficient. The nutrient uptake rate $f(c)$ per bacterium is expected to behave at low $c$ as $f(c) \sim k c$, with saturation at high $c: f(c) \sim k_{\max }$. When the nutrient is essential for life, such as oxygen for obligate aerobes, $f(c)$ may vanish below some $c^{*}$ [24,25]. As in Harper's study and Engelmann's experiment, the interesting regime has the resource limiting, with $b_{0}$ and $L$ such that the total nutrient flux can be consumed and is thus below the maximum value $k_{\max } b_{0} L^{d}$. Otherwise, steady state cannot be attained and $c$ increases indefinitely.
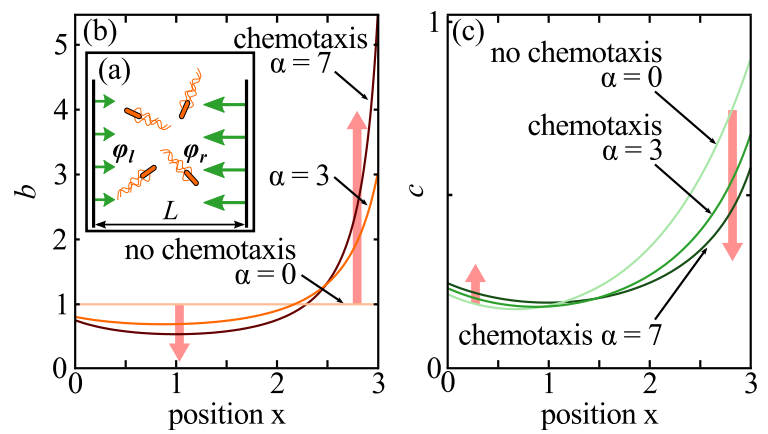

FIG. 1. (Color online) Numerical results in one dimension. (a) Sketch of the setup. (b) Example of a steady-state distribution of bacteria in the rescaled KS model, Eqs. (4) and (5). (c) Corresponding distribution of nutrient at steady state. In panels (b) and (c) the parameter values are $\lambda=3$ for the domain-size parameter and $s=1 / 8$ for the relative strength of the left source. The importance of chemotaxis against bacterial diffusion increases with the chemotactic parameter $\alpha$.

For small $c$, we identify the length scale $\ell_{k}=\left(D_{c} / k b_{0}\right)^{1 / 2}$ for concentration gradients due to uptake. For the a run-andtumble chemotaxis mechanism to operate and the continuum model to be relevant, $\ell_{k}$ should be large compared to the run length $\ell_{\text {run }}=v \tau$, where $v$ is a typical swimming speed and $\tau$ the time between tumbles. That is, we require the Knudsen-like number $l_{\text {run }} / l_{k} \ll 1$. For $E$. coli, with $v \sim 20 \mu \mathrm{m} / \mathrm{s}$ and $\tau \sim$ $1 \mathrm{~s}, \ell_{\text {run }} \sim 20 \mu \mathrm{m}$, but it can be considerably longer for other bacteria [28]. Still, at low $c$, with $\chi(c)=\chi_{0}$ [21], interesting behavior occurs when the chemotactic flux $\sim \chi_{0} b_{0} \phi_{0} / D_{c}$ dominates the diffusive flux $\sim D_{b} b_{0} / \ell_{k}$, with $\phi_{0} / D_{c}$ a typical concentration gradient. Thus, the Péclet-like number,

$$
\alpha=\frac{\chi_{0} \phi_{0}}{D_{b} D_{c} \ell_{k}},
$$

exceeds unity.

A one-dimensional version of Harper's experiment [Fig. 1(a)] has fluxes $\phi_{l}$ and $\phi_{r}$ at the left and right domain boundaries, and $\Phi=\phi_{l}+\phi_{r}$. Nondimensionalizing as above we obtain in the low- $c$ regime,

$$
\begin{gathered}
\frac{\partial c}{\partial t}=\nabla^{2} c-b c, \\
\delta^{-1} \frac{\partial b}{\partial t}=\nabla^{2} b-\alpha \nabla \cdot[b \nabla c],
\end{gathered}
$$

where $\delta=D_{b} / D_{c}$, with the following nondimensional boundary conditions:

$$
\begin{gathered}
\left.\hat{\mathbf{n}} \cdot \nabla c\right|_{0}=\left.s \quad \hat{\mathbf{n}} \cdot(\nabla b-\alpha b \nabla c)\right|_{0}=0, \\
\left.\hat{\mathbf{n}} \cdot \nabla c\right|_{\lambda}=1-\left.s \quad \hat{\mathbf{n}} \cdot(\nabla b-\alpha b \nabla c)\right|_{\lambda}=0,
\end{gathered}
$$

where $\hat{\mathbf{n}}$ is the outward unit normal to the domain.

Two new parameters drive the evolution of the system: $\lambda=L / \ell_{k}$, the domain size relative to the screening length, and the relative strength $s=\phi_{l} / \Phi$ of the left source. Before investigating the steady-state solutions to Eqs. (4) and (5), we point out that by construction the total uptake $U$ over the population is equal at steady state to the total nutrient flux into 
the chamber,

$$
U=\int_{0}^{\lambda} d x b c=\int_{0}^{\lambda} d x \nabla^{2} C=\left.\hat{\mathbf{n}} \cdot \nabla c\right|_{0} ^{\lambda}=1,
$$

independent of the choice of parameters and strength of chemotaxis. Thus, overall uptake optimization is not part of the present study: our interest resides instead in how the individual behaviors result in a distribution of finite resource among population members, just as in Harper's experiment.

One of the natural questions to ask is whether there is a variational structure to the KS Eqs. (4) and (5). The only related result of which we are aware concerns the case when the nutrient consumption rate $f(c)$ takes the aforementioned high- $c$ form of a constant. After suitable rescaling that dynamics is

$$
\begin{gathered}
\frac{\partial c}{\partial t}=\nabla^{2} c+v b, \\
\delta^{-1} \frac{\partial b}{\partial t}=\nabla^{2} b-\alpha \nabla \cdot[b \nabla c],
\end{gathered}
$$

where $v=-1$. As shown recently [26], a dynamics of a closely related form is variational. If we introduce the energy functional

$$
\mathcal{E}[b, c]=\int d \mathbf{x}\left\{\frac{1}{\alpha} b \log b-b c+\frac{1}{2}|\nabla c|^{2}\right\},
$$

then the variational relations,

$$
\frac{\partial c}{\partial t}=-\frac{\delta \mathcal{E}}{\delta c}, \quad \delta^{-1} \frac{\partial b}{\partial t}=\alpha \nabla \cdot\left[b \nabla \frac{\delta \mathcal{E}}{\delta b}\right],
$$

yield Eqs. (9) and (10), but with $v=+1$. This case corresponds to the well-studied situation in which the bacteria are sources for the chemoattractant, rather than sinks. Because the same term $(-b c)$ in $\mathcal{E}$ yields both the production and consumption term in Eq. (9) and the chemotactic term in Eq. (10), the case $v=-1$ appears not to have any variational structure of this type [27]. But since the consumption rate per bacterium is constant in this regime, the distribution of resource acquisition rates is trivial and a variational structure would provide no new information. More importantly, the case under consideration here, with $f(c) \sim c$, also appears not to possess a variational structure and thus it is not possible to conclude that the steadystate solutions are in any way minimizers or maximizers of some energy-like functional. It follows that the distribution of uptake rates is a nontrivial feature of the underlying diffusionconsumption-chemotaxis dynamics.

Figures 1(b) and 1(c) show steady-state distributions of $b$ and $c$ for $\alpha=3, \lambda=3, \quad \delta=0.6$, and $s=1 / 8$, which correspond to physical values of the dimensional parameters $\left(D_{c}=5 \times 10^{-6} \mathrm{~cm}^{2} \mathrm{~s}^{-1}\right.$, $D_{b}=3 \times 10^{-6} \mathrm{~cm}^{2} \mathrm{~s}^{-1}, k=2 \times 10^{-7}$ cell $^{-1} \mathrm{~s}^{-1} \mathrm{~cm}^{3}, \chi_{0}=$ $2.7 \times 10^{-3} \mathrm{~cm}^{2} \mathrm{~s}^{-1} \mathrm{mM}^{-1}, b_{0}=10^{6}$ cells cm ${ }^{-3}, \phi_{0}=3.4 \times$ $10^{-6} \mathrm{mM} \mathrm{cm} \mathrm{s}^{-1}$, and $\left.L=150 \mu \mathrm{m}[29,30]\right)$, along with the nonchemotactic case and the case $\alpha=7$. Bacteria accumulate on both sides, with more closer to the stronger source, as one might expect. Intriguingly, this leads to what we term chemotactic leveling of the nutrient: a more uniform concentration field than without chemotaxis. In particular, we notice that the maximum uptake rate of a bacterium in this population, obtained closest to the strongest source, is decreased by chemotaxis. Diffusion of $b$ and $c$ precludes the ideal free distribution.

\section{QUANTIFYING INEQUALITIES WITH THE GINI INDEX}

Whereas studies on biological consequences of chemotaxis usually measure the increased uptake over the whole or part of the population with respect to the nonchemotactic case (e.g., Refs. [31,32]), as emphasized above, the present study is focused on the case for which the mean uptake rate is independent of the chemotactic behavior. As our interest then resides in how equally resources are spread among the population, comparison of the chemotactic results with the nonchemotactic reference distributions thus requires a measure of the proximity with the ideal free distribution. Among the many possible measures of inequality [33], we consider here the Gini index $G \in[0,1]$ [22], which for a distribution $P(w)$ of wealth $w$ in a population can be expressed as

$$
G=\frac{\iint d u d v P(u) P(v)|u-v|}{2 \int d u u P(u)} .
$$

The ideal free distribution, in which every individual has the same wealth $u_{0}$, is $P=\delta\left(u-u_{0}\right)$, and thus $G=0$, while larger values hold for more unequal distributions. $G$ can be used with any notion of "wealth" [34], such as biodiversity [35]. We should empasize that in using the Gini index to quantify uptake inequalities in the present study we do not imply any preferred status to $G$ as a metric for resource acquisition distributions. There are many that could be explored, the Gini index presenting the advantage of being easily translated in the context of the continuous distribution and thus enabling analytical work on its variations. Using the individual nutrient uptake rate as wealth, we can transform the integrals in the uptake rate levels into integrals in space. This makes the bacterial density appear as the equivalent in space of the frequency distribution of uptake levels. Normalizing this new density function, thus making the integral for the total number of bacterial cells appear at the denominator, we finally re-express Eq. (13) for time-dependent spatial distributions in a domain $\Omega$ :

$$
G(t)=\frac{\int_{\Omega} \int_{\Omega} d x d y b(\mathbf{x}, t) b(\mathbf{y}, t)|f(c(\mathbf{x}, t))-f(c(\mathbf{y}, t))|}{2 \int_{\Omega} d x b(\mathbf{x}, t) f(c(\mathbf{x}, t)) \int_{\Omega} d x b(\mathbf{x}, t)} .
$$

With this measure of inequality, we investigate how the uptake distribution at steady state depends on the parameters of the KS model Eqs. (4) and (5). Before proceeding we should make clear that the Gini index values calculated within the present approach and the corresponding inequalities in the uptake levels are instantaneous: physically, bacteria would swim along a biased random walk inside the steady-state nutrient distribution, thus sampling different concentration levels. Over a time long in comparison with the typical time of bacterial diffusion at the scale of the experimental chamber this motility would tend to level the integrated uptake within the population and yield lower values of $G$. The fundamental issue is then whether the timescale for this smoothing-out of inequalities is large or small compared to the timescale $\tau_{\text {int }}$ for a relevant internal biological process based on nutrient uptake. 


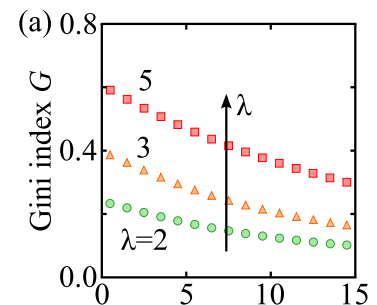

chemotactic parameter $\alpha$

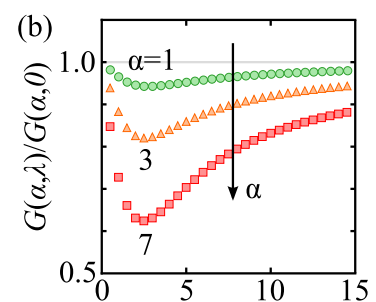

domain size parameter $\lambda$

FIG. 2. (Color online) Variations of Gini index for bacterial uptake, with $s=0$ (single source on the right side). (a) $G$ at steady-state with respect to the chemotactic parameter $\alpha$ for various domain sizes $\lambda$. (b) Ratio of $G$ in the chemotactic case to that without, for various chemotactic strengths $\alpha$, showing optimal domain size parameter for relative decrease of $G$.

The present approach is thus valid in the limit $\tau_{\text {int }} D_{b} / L^{2} \ll 1$, thus most relevant to large system sizes and short internal times. As an example, it takes approximately $17 \mathrm{~h}$ for a typical run-and-tumble bacteria $\left(D_{b}=4 \cdot 10^{-6} \mathrm{~cm}^{2} \cdot \mathrm{s}^{-1}\right)$ to explore the space between two sources separated by $5 \mathrm{~mm}$, a time that is much bigger than the typical scale of key cellular processes such as division (approximately $30 \mathrm{~min}$ ).

We now go back to investigating the role of each parameter: the ratio of diffusion coefficients $\delta$ impacts transient dynamics but does not modify steady-state solutions. From numerical solutions in the phase space delimited by $s \in[0,0.5]$ (from one source on the right to equal sources), $\alpha \in[0,15]$ (strength of chemotaxis) and $\lambda \in[0.5,15]$ (domain size), we obtain first the intuitive result that for given chemotactic and domain-size parameters, the more equal the sources are, the more equal the uptake is among the population, and the lower is $G$. More balanced sources indeed create smaller nutrient gradients, thus a lesser range of uptakes and weaker chemotaxis. We also find that $G$ increases with the size parameter $\lambda$ [Fig. 2(a) in the case $s=0$ ], for larger $\lambda$ corresponds to stronger variations of the concentration field and higher variations of uptake.

The question of whether chemotactic leveling of the nutrient concentration field can make the distribution of individual uptake more ideal is addressed by varying $\alpha$. Its impact is subtle: chemotaxis levels nutrients across the domain, but accumulation of cells near sources improves uptake of some to the detriment of others. We find that $G$ actually decreases with $\alpha$ [Fig. 2(a) for a single source], which reveals chemotactic leveling of the uptake rate among the population. In Fig. 1, $G$ decreases from $\simeq 0.3$ with no chemotaxis to $\simeq 0.25$ when chemotaxis $(\alpha=3)$ is allowed.

This decrease is best understood for a single source on one side of the domain. Because $c$ decreases monotonically from the source, the bacterial population can be split into quantiles of uptake that are ordered in space. Chemotaxis lowers the nutrient concentration over the whole domain, but mostly close to the source, and it shifts the center of mass and mean uptake level of each quantile. Figure 3(a) shows that bacteria with the higher uptake, which are also closer to the source, are transferred to lower uptake levels. For the lower uptake quantiles, higher levels of uptake compared to the nonchemotactic case are attained due to chemotaxis toward the source. Together, these effects bring uptake levels closer

(a)

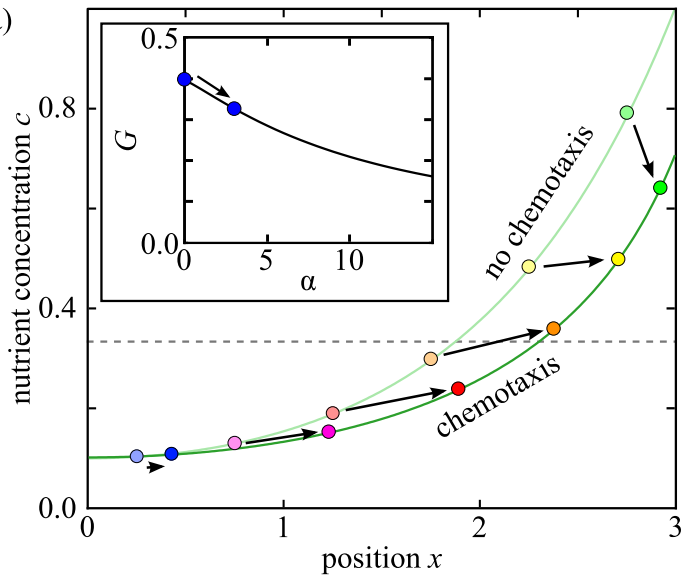

(b)

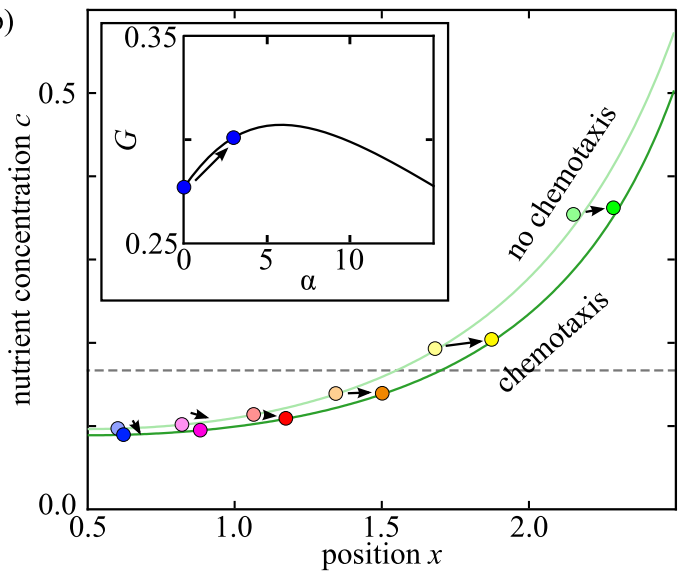

FIG. 3. (Color online) Interpretation of steady-state Gini index variation in different dimensions $d$. (a) $d=1$ with domain-size parameter $\lambda=3, s=0$ (single source on right side), chemotacticstrength parameter $\alpha=3$. (b) $d=2$ with a circular source of size $l=0.5$ in a domain of radius $L=4.5$, and chemotactic-strength parameter $\alpha=3$. Shown are center of mass and mean uptake level of the sextiles of uptake distribution (colored circles), overlaid on nutrient concentration, without and with chemotaxis. Dashed line shows concentration for average uptake. Inset: $G$ vs. chemotacticstrength parameter $\alpha$, with blue circles corresponding to displayed solutions.

to the average, lowering $G$ : the bacterial system moves closer to the ideal free distribution.

The generality of this result can be established in the limit of weak chemotaxis $(\alpha \ll 1)$ still with a single source, where a series solution $G \simeq G_{0}(\lambda)+\alpha G_{1}(\lambda)+\cdots$ of Eqs. (4) and (5) yields

$$
\begin{gathered}
G_{0}(\lambda)=1-2 \frac{\cosh \lambda-1}{\lambda \sinh \lambda}, \\
G_{1}(\lambda)=-\frac{2}{3 \lambda}+\frac{\cosh \lambda-1}{\lambda^{2} \sinh \lambda}\left[1+\frac{\lambda \cosh \lambda}{3 \sinh (\lambda)}\right],
\end{gathered}
$$

where $G_{1}(\lambda)<0$, so $G$ indeed decreases with chemotaxis. A cumbersome analysis (not shown) for the general case $s \in$ $[0,0.5]$, that is $G \simeq G_{0}(\lambda, s)+\alpha G_{1}(\lambda, s)+\cdots$, also shows that $G_{1}(\lambda, s)<0$, thus extending this result to any ratio of source strengths. 
In the limit of strong chemotaxis $(\alpha \gg 1)$, where bacterial diffusion becomes irrelevant, we may expect to recover the ideal free distribution. Analytical progress on this steady-state problem is achieved by integrating twice Eq. (5) to obtain $b(c)$, substituting into Eq. (4) and then expanding in powers of $1 / \alpha$. One obtains

$$
\begin{gathered}
b(x)=\frac{1}{\alpha} \frac{\lambda \omega^{2}}{2 \cos ^{2}\left[\omega\left(x-\beta_{1}\right) / 2\right]}, \\
c(x)=\frac{1}{\lambda}+\frac{1}{\alpha}\left(\beta_{2}-2 \log \left\{\cos \left[\omega\left(x-\beta_{1}\right) / 2\right]\right\}\right),
\end{gathered}
$$

where $\omega$ and $\beta_{1}$ are determined through

$$
\begin{gathered}
\omega \tan \left(\frac{\omega}{2} \beta_{1}\right)=\alpha s, \\
\omega \tan \left[\frac{\omega}{2}\left(-\beta_{1}+\lambda\right)\right]=\alpha(1-s) .
\end{gathered}
$$

The constant $\beta_{2}$ depends on $\omega$ and the model parameters. Neglecting terms in $1 / \alpha^{2}$ and higher order, it can be written as

$$
\begin{aligned}
\beta_{2} \simeq & -2 \log \alpha+2+2 \log \omega \\
& -2[s \log s+(1-s) \log (1-s)]-\omega^{2} \lambda / \alpha+\cdots .
\end{aligned}
$$

This solution enables us to obtain an analytical expression for the Gini index in the limit of strong chemotaxis:

$$
G \simeq \lambda\left\{1-2 s+4 s^{2}+s(1-s) \log \left[\frac{(1-s)^{2}}{s^{2}}\right]\right\} \frac{1}{\alpha}+\cdots,
$$

to leading order in $1 / \alpha$, for $s \in[0,0.5]$. This establishes further the generality of its decrease with $\alpha$ together with its increase with $\lambda$ : in $1 \mathrm{D}$, chemotaxis levels the uptake throughout the population. Moreover, in this range of high $\alpha$, inequalities of uptake initially increase when the system changes from a single source to more balanced sources.

As this leveling of the uptake distribution appears as the microbial equivalent of the more uniform uptake displayed by ducks, it is natural to ask if, as in Harper's experiments, the bacterial population reaches the ideal free distribution as $\alpha \rightarrow \infty$ and splits into two localized subpopulations proportional to the source strengths. If we consider that the position $\beta_{1}$ of minimum bacterial concentration separates a left population $B_{L}$ associated to the left source and its right-hand-side equivalent $B_{R}$, our analytical solution for $\alpha \gg 1$ directly yields

$$
\int_{0}^{\beta_{1}} d x b(x)=B_{L} \simeq \lambda s .
$$

Thus, the population associated to one source is directly proportional, to leading order, to the flux of this source, as in the ideal free distribution. Moreover, analysis of Eq. (17) shows that in the limit $\alpha \rightarrow \infty, b(x)$ is localized in regions of width $\sim 1 / \sqrt{\alpha}$ at both $x=0$ and $x=\lambda$, with peak values $\sim \alpha$ at these positions. In the limit of $\alpha \rightarrow \infty$, we thus get a localization of the number of cells proportional to the source at the source: this is the (unphysical) limit of a microbial ideal free distribution.

When the domain-size parameter $\lambda \gg 1$, the central portion of the domain has a steady-state concentration $c \sim 0$, with very small gradients. Bacteria there are screened from the sources, unable to feel sufficient gradients to move chemotactically closer to them. The relative redistributive effect of chemotaxis compared to its absence must then diminish with distance. Considering the relative Gini index $G(\alpha) / G_{0}$ in the approximation Eqs. (15) and (16), we indeed find an optimal domain size, $\lambda_{G} \simeq 3.12$, for which the redistributive effect of chemotaxis is the strongest. An optimal size is also found in simulations beyond the linear regime in $\alpha$ [Fig. 2(b)] and with influx from both sides, with $\lambda_{G} \simeq 3$. The decrease of this relative change for high values of $\lambda$ embodies the aforementioned screening, while the behavior at low $\lambda$ results from a nearly uniform concentration over the domain, with only weak gradients for a chemotactic response.

Does the uptake leveling found in $d=1$ hold in higher dimensions? To answer this, we solve Eqs. (4) and (5) for a single spherical source of radius $l$ in a closed spherical domain of radius $L$, both measured in units of $l_{k}$. We find that in $d=2$ and 3 the effects of chemotaxis, for a given size of source and domain, are much weaker. Moreover, for certain parameter values, chemotaxis can actually increase $G$ [inset in Fig. 3(b)]. Analysis of the quantiles [Fig. 3(b) for a two-dimensional example] shows that in these cases, even though bacteria closest to the source have a lowered uptake, a majority of the bacteria that are already above the average uptake in the nonchemotactic case gain access to even higher uptake. Bacteria furthest from the source, and below the average uptake level in the nonchemotactic case, see their mean uptake decrease even further. Overall this corresponds to an increase of $G$ : in higher dimensions, chemotaxis can bring the bacteria further away from the ideal free distribution, that is, it increases the inequalities among the population. The increase or decrease of inequalities of uptake, as revealed by the positive or negative change of $G$, may thus depend in detail on the system characteristics embodied in $l, L$, and $\alpha$.

\section{IMPLICATIONS FOR FITNESS}

What would be the biological consequences of chemotactic leveling of resources and of uptake rates? Uptake of nutrients governs a wide range of bacteriological processes, among which are cell growth and division. In particular, the yield of biomass per unit nutrient taken up is an increasing function of the available nutrient concentration, a feature that has been suggested as selective for the response characteristics of chemotaxis [13]. Here we provide a brief discussion of how the chemotactically driven redistribution of resources throughout a population can impact on the average growth rate of the population, which we consider a measure of fitness. Continuing the point of view taken in the introduction, we show that while at the single-cell level in a defined resource field chemotaxis may increase fitness, this is not necessarily true at the population level.

We compute an average growth rate $\bar{\mu}$ over the population from the steady-state distributions of Eq. (5), in a model in which the local growth rate $\mu(x)$ is proportional to the local uptake rate through a yield function $y(c): \mu(x)=y(c) c$ and

$$
\bar{\mu}=\frac{1}{\lambda} \int_{0}^{\lambda} d x y(c) c b .
$$




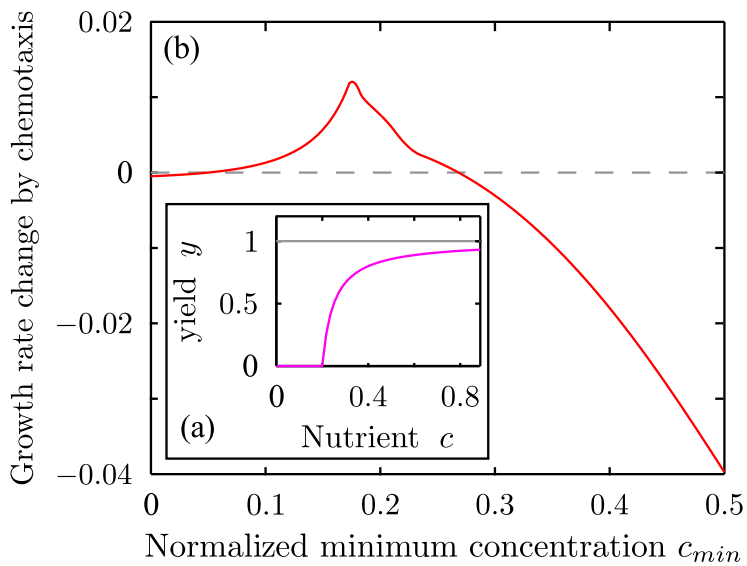

FIG. 4. (Color online) Consequences of uptake levelling on population growth rate. Using $\alpha=4, \lambda=3$, and $s=0.125$ in $d=1$. (a) Yield as a function of nutrient concentration, for $c_{\min }=0.2$, $K=0.05$, and $y_{0}=1$. (b) Change of growth rate as a consequence of chemotaxis for $K=0.05$ and varying $c_{\min }$ : if chemotaxis can increase growth for low-yield threshold, the uptake leveling makes it less advantageous for higher threshold by diluting the resource.

Whereas the mean uptake is fixed by the boundary conditions in our problem, this average-growth coefficient will depend on the distribution of the resource among the bacteria and thus will be modified by chemotaxis. In order to capture the increase of $y$ with $c$, we consider that $y=0$ below a threshold concentration $c_{\min }$, and adopt a Michaelis-Menten form above it [37]:

$$
y(c)=y_{0} \frac{\left(c-c_{\min }\right)}{\left(c-c_{\min }\right)+K} \quad \text { for } \quad c>c_{\min },
$$

with $K$ a saturation constant of the yield [Fig. 4(a)].

The threshold concentration $c_{\text {min }}$ can be considered as the limit below which all the uptake is directed toward maintenance costs [36]. The relative change of the average growth rate due to chemotaxis ( $\bar{\mu}_{\text {chemo }}-\bar{\mu}_{\text {non chemo }}$ ) is shown in Fig. 4(b). We observe that for lower values of the threshold concentration $c_{\text {min }}$ the redistributive effect increases the number of cells that reach the growth threshold: the population fitness becomes higher with chemotaxis than without. However, for higher values of the threshold concentration $c_{\text {min }}$, the redistribution of uptake throughout the population leaves more cells below the growth threshold: we get the counterintuitive result that chemotaxis effectively lowers population fitness with respect to the nonchemotactic case. This result, which stems from the competition of the bacteria for the same resource, shows that in the context of continuous sources, an homogeneously chemotactic population could be selected against due to the dilution of a scarce resource resulting from the chemotactic behavior.

\section{CONCLUSIONS}

We have shown that the organization of bacteria around localized nutrient sources is fundamentally different from that of higher animals due to diffusion of resources and feeders. Yet, there are still common characteristics. First, what might be termed "foraging" behavior decreases the maximum uptake rate through competition for the resource; in the bacterial case this corresponds to a decrease of the maximum of the concentration field with chemotaxis. Second, foraging generates, quite evidently, localization and accumulation of the population closer to the resources. But whereas the conjunction of these two phenomena brings Harper's ducks to the ideal free distribution, it may fail in the microbial world: it brings the system closer or further from this ideal distribution depending on the spatial dimensionality and parameters capturing the strength of chemotaxis, the size of the resources, and the distance between them. The redistribution of uptake is not without consequences: when the resource is scarce in comparison to the metabolic needs, chemotaxis effectively dilutes it and reduces the average population fitness.

The issues addressed here suggest experimental studies of model systems in physical ecology for which in situ measurements of local metabolic activity and nutrient concentration fields are possible. Optically based quantitative measures of photosynthetic activity [38], probes of local oxygen concentration [39], and local mass spectrometry [40] are examples of relevant techniques. Microbial communities in biofilms, sediments [41], and algae sustaining a motile population of bacteria around them by releasing oxygen [42] represent interesting systems in which to study the distribution of uptake rates.

\section{ACKNOWLEDGMENTS}

We are grateful to Wolfram Schultz for bringing Ref. [1] to our attention, and thank O. Croze, T.J. Pedley, W. Poon, A. Smith, G. Peng, and R. Watteaux for discussions. This work was supported by a Raymond and Beverly Sackler Scholarship (F.J.P.), MinesParisTech (F.J.P.), and the European Research Council Advanced Investigator Grant No. 247333 (R.E.G.).
[1] D. G. C. Harper, Competitive foraging in mallards: "Ideal free" ducks, Anim. Behav. 30, 575 (1982).

[2] S. D. Fretwell and H. L. Lucas Jr., On territorial behavior and other factors influencing habitat distribution in birds, Acta Biotheor. 19, 16 (1969).

[3] P. Glimcher, M. Dorris, and H. Bayer, Physiological utility theory and the neuroeconomics of choice, Games Econ. Behav. 52, 213 (2005).
[4] M. Kennedy and R. D. Gray, Can ecological theory predict the distribution of foraging animals? A critical analysis of experiments on the ideal free distribution, Oikos 68, 158 (1993).

[5] M. L. Rosenzweig, A theory of habitat selection, Ecology 62, 327 (1981).

[6] J. K. Parrish and L. Edelstein-Keshet, Complexity, pattern, and evolutionary trade-offs in animal aggregation, Science 284, 99 (1999). 
[7] G. J. Madden, B. F. Peden, and T. Yamaguchi, Human group choice: Discrete-trial and free-operant tests of the ideal free distribution, J. Exp. Anal. Behavior 78, 1 (2002).

[8] K. E. Abernethy, E. H. Allison, P. P. Molloy, and I. M. Côté, Why do fishers fish where they fish? Using the ideal free distribution to understand the behavior of artisanal reef fishers, Can. J. Fish. Aquat. Sci. 64, 1595 (2007).

[9] J. R. Kraft, W. M. Baum, and M. J. Burge, Group choice and individual choices: Modeling human social behavior with the ideal free distribution, Behav. Process. 57, 227 (2002).

[10] F. Azam and F. Malfatti, Microbial structuring of marine ecosystems, Nat. Rev. Microbiol. 5, 782 (2007).

[11] H. C. Berg, Random Walks in Biology, 2nd ed. (Princeton University Press, Princeton, NJ, 1993).

[12] H. C. Berg and E. M. Purcell, Physics of chemoreception, Biophys. J. 20, 193 (1977).

[13] A. Celani and M. Vergassola, Bacterial strategies for chemotaxis response, Proc. Natl. Acad. Sci. U.S.A. 107, 1391 (2010).

[14] T. W. Engelmann, Untersuchungen über die quantitativen Beziehungen zwischen Absorption des Lichtes und Assimilation in Pflanzenzelle, Bot. Zeit. 42, 81 (1884).

[15] T. W. Engelmann, Neue Methode zur Untersuchung der Sauerstoffausscheidung pflanzlicher und thierischer Organismen, Pflüg. Arch. Ges. Phys. 25, 285 (1881).

[16] M. T. Croft, A. D. Lawrence, E. Raux-Deery, M. J. Warren, and A. G. Smith, Algae acquire vitamin B12 through a symbiotic relationship with bacteria, Nature 438, 90 (2005).

[17] N. Blackburn, T. Fenchel, and J. Mitchell, Microscale nutrient patches in planktonic habitats shown by chemotactic bacteria, Science 282, 2254 (1998).

[18] T. Kiørboe and G. A. Jackson, Marine snow, organic solute plumes, and optimal chemosensory behavior of bacteria, Limnol. Oceanogr. 46, 1309 (2001).

[19] E. F. Keller and L. A. Segel, Traveling bands of chemotactic bacteria: A theoretical analysis, J. Theor. Biol. 30, 235 (1971).

[20] J. D. Murray, Mathematical Biology II Spatial Models and Biomedical Applications, 3rd ed. (Springer-Verlag, New York, 2003).

[21] M. Tindall, P. Maini, S. Porter, and J. Armitage, Overview of mathematical approaches used to model bacterial chemotaxis II: Bacterial populations, Bull. Math. Biol. 70, 1570 (2008).

[22] C. Gini, Variabilità e mutabilità (Bologna, C. Cuppini, 1912).

[23] M. O. Lavrentovich, J. H. Koschwanez, and D. R. Nelson, Nutrient shielding in clusters of cells, Phys. Rev. E 87, 062703 (2013).

[24] A. J. Hillesdon, T. J. Pedley, and J. O. Kessler, The development of concentration gradients in a suspension of chemotactic bacteria, B. Math. Biol. 57, 299 (1995).

[25] I. Tuval, L. Cisneros, C. Dombrowski, C. W. Wolgemuth, J. O. Kessler, and R. E. Goldstein, Bacterial swimming and oxygen transport near contact lines, Proc. Natl. Acad. Sci. USA 102, 2277 (2005).

[26] See, for example, A. Blanchet, J. A. Carrillo, D. Kinderlehrer, M. Kowalczyk, P. Laurencot, and S. Lisini, A hybrid variational principle for the Keller-Segel System in $\mathbb{R}^{2}$, arXiv:1407.5562.

[27] See also G. Wolansky, Multi-components chemotactic system in the absence of conflicts, Euro. J. App. Math. 13, 641 (2002); A critical parabolic estimate and applications to nonlocal equations arising in chemotaxis, App. Anal. 66, 291 (1997).

[28] J. G. Mitchell, M. M. Martinez-Alonso, J. Lalucat, I. Esteve and S. Brown, Velocity changes, long runs, and reversals in the chromatium minus swimming response, J. Bacteriol. 173, 997 (1991).

[29] A. Natarajan and F. Srienc, Dynamics of glucose uptake by single Escherichia coli cells, Metab. Eng. 1, 320 (1999).

[30] T. Ahmed and R. Stocker, Experimental verification of the behavioral foundation of bacterial transport parameters using microfluidics, Biophys. J. 95, 4481 (2008).

[31] R. Stocker, J. Seymour, A. Samadani, D. Hunt, and M. Polz, Rapid chemotactic response enables marine bacteria to exploit ephemeral microscale nutrient patches, Proc. Natl. Acad. Sci. USA 105, 4209 (2008)

[32] J. Taylor, and R. Stocker, Trade-offs of chemotactic foraging in turbulent water, Science 338, 675 (2012).

[33] F. Cowell, Measuring Inequality (Oxford University Press, Oxford, 2011).

[34] C. Gini, Measurement of inequality of incomes, Econ. J. 31, 124 (1921).

[35] L. Wittebolle et al., Initial community evenness favours functionality under selective stress, Nature 458, 623 (2009).

[36] D. Tempest, The biochemical significance of microbial growth yields: A reassessment, Trends Biochem. Sci. 3, 180 (1978).

[37] H. L. Smith and P. Waltman, The Theory of the Chemostat: Dynamics of Microbial Competition, Cambridge Studies in Mathematical Biology, Vol. 13 (Cambridge University Press, Cambridge, UK, 1995).

[38] N. R. Baker, Chlorophyll fluorescence: A probe of photosynthesis in vivo, Annu. Rev. Plant Biol. 59, 89 (2008).

[39] C. Douarche, A. Buguin, H. Salman, and A. Libchaber, E. coli and oxygen: A motility transition, Phys. Rev. Lett. 102, 198101 (2009).

[40] J. D. Watrous and P. C. Dorrestein, Imaging mass spectrometry in microbiology, Nat. Rev. Microbiol. 9, 683 (2011).

[41] R. N. Glud et al., An in situ instrument for planar O2 optode measurements at benthic interfaces, Limnol. Oceanogr. 46, 2073 (2001).

[42] J. Adler and B. Templeton, The effect of environmental conditions on the motility of Escherichia coli, J. Gen. Microbiol. 46, 175 (1967) 